Arnold Schönberg - Das Leben im Werk 
Alexander L. Ringer

\section{Arnold Schönberg \\ Das Leben im Werk}

Mit einem Nachwort von Thomas Emmerig

Verlag J.B. Metzler Stuttgart · Weimar

Bärenreiter Kassel 
Gemeinschaftsausgabe der Verlage J. B. Metzler, Stuttgart und Bärenreiter, Kassel

Den allzu früh verstorbenen Freunden

Carl Dahlhaus und Alan P. Lessem

zum Andenken

Die Deutsche Bibliothek - CIP-Einheitsaufnahme

Ringer, Alexander L.:

Arnold Schönberg : das Leben im Werk / Alexander L. Ringer.

Mit einem Nachw. von Thomas Emmerig.

Stuttgart ; Weimar : Metzler; Kassel : Bärenreiter, 2002

ISBN 978-3-476-01906-6

ISBN 978-3-476-01906-6 (J.B. Metzler)

ISBN 978-3-476-02864-8 (eBook)

DOI 10.1007/978-3-476-02864-8

ISBN 978-3-7618-2029-2 (Bärenreiter)

Dieses Werk einschließlich aller seiner Teile ist urheberrechtlich geschützt. Jede Verwertung außerhalb der engen Grenzen des Urheberrechtsgesetzes ist ohne Zustimmung des Verlages unzulässig und strafbar. Das gilt insbesondere für Vervielfältigungen, Übersetzungen, Mikroverfilmungen und die Einspeicherung und Verarbeitung in elektronischen Systemen.

(C) 2002 Springer-Verlag GmbH Deutschland

Ursprünglich erschienen bei J.B. Metzlersche Verlagsbuchhandlung

und Carl Ernst Poeschel Verlag GmbH in Stuttgart 2002 


\section{Inhalt}

Vorwort $\ldots \ldots \ldots \ldots \ldots \ldots \ldots \ldots \ldots \ldots \ldots \ldots \ldots \ldots \ldots$, VII

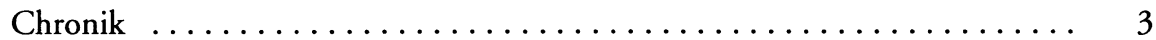

Zur Einleitung: Ein österreichisch-jüdisches Künstlerschicksal $\ldots \ldots \ldots \ldots \quad 65$

Lehre als Sozialreform ........................... 77

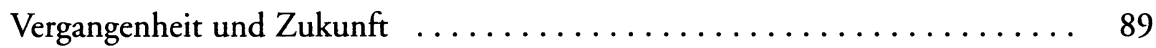

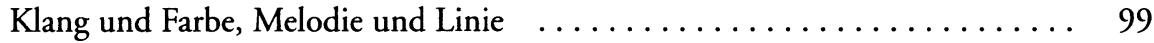

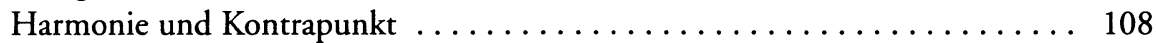

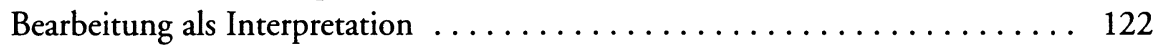

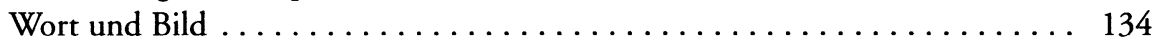

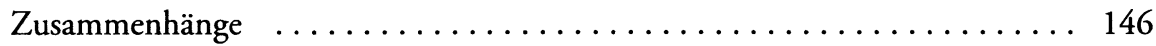

Ein „Drama mit Musik“ und „Dreimal sieben Melodramen“ $\ldots \ldots \ldots \ldots .168$

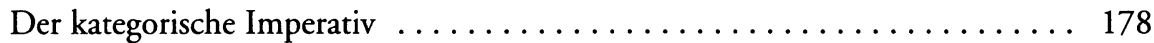

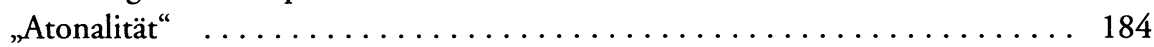

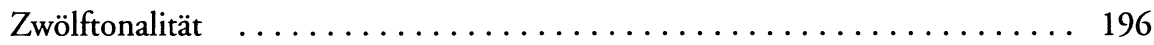

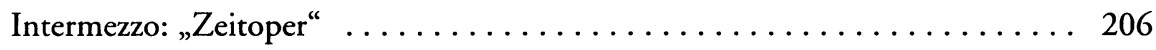

Instrumentalkomposition "mit zwölf Tönen" $\ldots \ldots \ldots \ldots \ldots \ldots \ldots \ldots . \ldots \ldots$

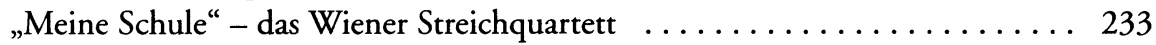

Zwölftonlehre: das Ei des Kolumbus? . . . . . . . . . . . . . . . 241

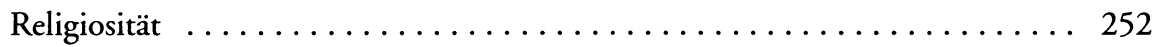

Rückkehr in die Zukunft („Ma fin est mon commencement") $\ldots \ldots \ldots \ldots 266$

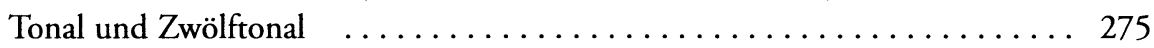

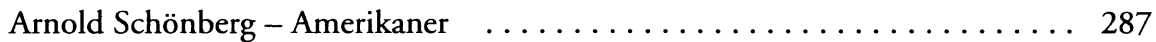

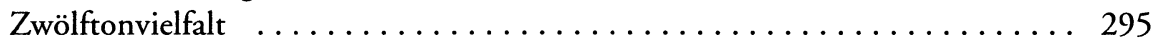

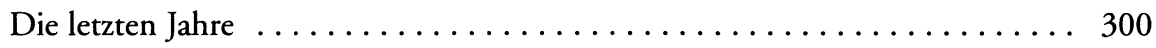

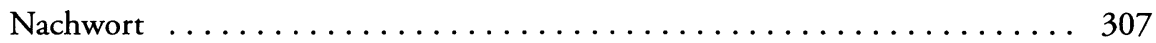

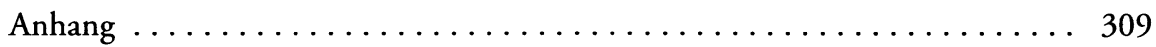

Chronologisches Werkverzeichnis ......................... 309

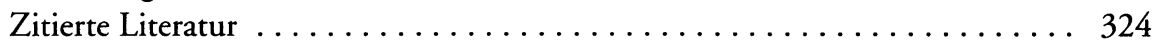

Register der zitierten Werke Arnold Schönbergs $\ldots . . . \ldots \ldots . . . . . .329$

Register ..................................... 331

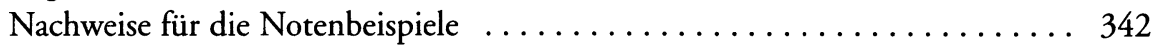




\section{Vorwort}

Arnold Schönberg war seine Zeit. Denn dieser Komponist oder vielmehr dieser auf zahlreichen Gebieten tätige Künstler spiegelte in seinem Leben, in seinem Werk die Zeit so genau und vielfältig wider, daß sein Schaffen zumindest in psychologischer und soziologischer Hinsicht eine Geschichte der ersten Hälfte des 20. Jahrhunderts darstellt. Aus dieser unleugbaren Tatsache ergeben sich für den Chronisten zahlreiche Probleme, die der vorliegende Band ebensowenig zu lösen vermochte wie die inzwischen umfangreiche Schönberg-Literatur im allgemeinen.

Da ist zunächst das Schicksal des Menschen Schönberg, der - als Jude geboren, später evangelisch getauft, dann offiziell zum Judentum zurückgekehrt - seinem jüdischen Schicksal nicht zu entgehen vermochte, ja eigentlich nie zu entgehen versuchte. Es ist ein Thema, dem sich der Autor verhältnismäßig ausführlich in einigen seiner englischen Studien gewidmet hat, das im übrigen auch einen weitaus jüngeren, den allzu früh verschiedenen deutschen Musikwissenschaftler Michael Mäckelmann, beschäftigte. Obwohl es sich ganz offensichtlich erübrigte, in diesem Zusammenhang noch einmal auf Einzelheiten einzugehen, schien es dennoch unerläßlich, hier wenigstens eine resümierende Zusammenfassung vorzulegen. Andererseits bot eine verhältnismäßig ausführliche Chronik die Gelegenheit, wichtige Daten politischer Ereignisse sowie literarischer und anderer künstlerischer Entwicklungen in Erinnerung zu bringen. Das Resultat war allerdings nicht nur ein weitaus größerer Umfang als der einer nur kurz orientierenden Chronik, sondern auch eine umfassendere Skizzierung eng miteinander verbundener Ereignisse, die für das Verständnis von Schönbergs Werdegang von grundsätzlicher Bedeutung erschienen. Dieser Überblick sollte dem interessierten Leser zumindest den nötigen Rahmen für ein besseres Verständnis der folgenden Kapitel vermitteln, gehen sie doch im Fall Schönbergs weit über das hinaus, was man im allgemeinen von einem Komponisten erwarten würde.

In diesem Buch ist daher verhältnismäßig viel von Dichtung und Malerei, von Religion und Pädagogik und anderen Interessen die Rede, die dem Komponisten Schönberg eng am Herzen lagen und einen großen Teil seines musikalischen Schaffens bestimmten. Es gab kaum ein geistiges Gebiet, mit dem er sich nicht beschäftigte, was ihn jedoch nicht hinderte, auch handwerkliche und sonstige praktische Interessen zu verfolgen. Man denke nur daran, daß er, ein Freund des Wiener Architekten Adolf Loos, unter dem Eindruck von aus der Wiener Werkstatt hervorgegangenen Arbeiten alle möglichen Dinge entwarf und sogar selbst anfertigte - u. a. Möbel, Spielkarten, Straßenbahnfahrscheine und Notenschreibmaschinen. Kurzum, man wäre versucht, von einem Universalgenie zu sprechen, wenn das nicht eine allzu oft mißbrauchte Bezeichnung wäre. Bei seiner Ankunft in den Vereinigten Staaten 1933 wurde er jedenfalls allgemein als „Einstein der Musik“ begrüßt.

Die weitaus größte Anzahl von Arbeiten über Arnold Schönberg befaßt sich mit analytischen Problemen, insbesondere in Bezug auf die von ihm entwickelte und 
in seinen reifen Jahren zumeist angewendete Methode, mit zwölfTönen zu komponieren. Schönberg selbst hatte für derartige Studien äußerst wenig übrig. Mit „Notenzählern" wollte er nichts zu tun haben. Und wie er sich seinem Schwager und hervorragenden Interpreten, dem Geiger und Quartettprimarius Rudolf Kolisch, gegenüber einmal beschwerte, sollte man doch endlich aufhören, immer wieder auf das "Wie“ statt auf das „Was" seiner Musik einzugehen. Die hier vorliegende Studie gibt denn auch dem „Was“ den Vorrang und statt dem „Wie“ dem „Wieso" oder „Warum“. Mit anderen Worten, es handelt sich um einen Versuch, so weit wie möglich Verbindungen herzustellen, Verbindungen kompositorischer Entwicklungen mit Vorgängen auf anderen Gebieten einerseits und andererseits verschiedener Werke untereinander.

Schönberg betonte immer wieder die Logik seiner Arbeiten und lehrte unermüdlich den Grundsatz, daß aus einem guten Gedanken alles weitere hervorgeht. Sein Ideal war die Einheit alles Menschlichen im Ebenbild der Einheit Gottes, die der gläubige Jude in jedem seiner Gebete mehrere Male am Tage zum Ausdruck bringt. Im Interesse der Einheit werden dem aufmerksamen Leser daher absichtliche Wiederholungen oder vielmehr Varianten von bereits Ausgedrücktem sowie Rückbezüge begegnen, die an und für sich schon einer Interpretation des Schönbergschen Denkens und seiner Arbeitsweise gleichkommen. Nicht jedem werden sie so natürlich erscheinen wie dem Autor, der seinerseits auf den Segen des großen künstlerischen Geistes hofft, von dem hier die Rede ist.

Wie manche Arbeit des Subjekts dieser Studie erstreckte sich ihre Vollendung über viele Jahre, ja selbst Jahrzehnte. Daß sich aus diesem unvermeidlichem Umstand gewisse Schwierigkeiten ergaben, bedarf keiner besonderen Betonung. Andererseits aber hofft der Autor, daß in der langen Zwischenzeit gewonnene Einsichten dem Buch eher zugute gekommen sind. Denn Arnold Schönberg glich seinem Idol Gustav Mahler auch insofern, als spätere Generationen der Gefahr ausgesetzt sind, sich ihm ausschließlich vom Standpunkt ihrer eigenen Zeit zu nähern, während er eigentlich nur im Geist seiner Zeit, ihrer Probleme und Ideale, greifbar wird. Aber ob man in ihm nun den letzten Romantiker sieht, den „konservativen Revolutionär" oder den fortschrittlich gesinnten Vater der „Neuen Musik“, seine Lebenszeit umspannte eine in der Geschichte fast einmalig dastehende Epoche, in der sich die Lebensbedingungen und Aspirationen von Millionen von Menschen derart drastisch veränderten, daß ihr Verhältnis zur Vergangenheit so gut wie unüberbrückbaren Schwierigkeiten unterliegt. Zumindest in dieser Beziehung hat der Schönberg-Biograph einen nicht zu unterschätzenden Vorteil, wenn er selbst kein junger Mann mehr ist. Wie gesagt handelt es sich im vorliegenden Fall nicht um eine Biographie im gewohnten Sinn des Wortes, wenn überhaupt, dann eher im Sinn einer musikalischen Biographie aus der Perspektive des Komponisten.

Selbstverständlich trägt der Autor die Verantwortung für den Inhalt und die Formulierungen eines Buches, das ihm, der seit einem halben Jahrhundert kaum noch Gelegenheit hat, deutsch zu sprechen, keinesfalls leicht gefallen ist. Für sein Zustandekommen ist der Autor Dr. Thomas Emmerig zu Dank verpflichtet, dessen wachsamem Auge keine Unstimmigkeit entgangen ist, und der ihm auch sonst mit Rat und Tat zur Seite gestanden hat. Hinsichtlich der dem Buch zugrundeliegenden Dokumente hat sich Wayne Shoat, der ehemalige Archivar des Arnold-Schönberg-Instituts in Los 
Angeles, ebenso wie Steven Whiting durch die Materialsammlung für die Chronik besonders verdient gemacht. Ohne die stets freundliche Hilfe des Personals der Musikbibliothek an der University of Illinois gäbe es andererseits kaum Fußnoten. Und ohne die unentwegte Unterstützung meiner so lange Zeit geduldig wartenden Frau wäre überhaupt nichts zustande gekommen.

Frankfurt, 17. April 2002

Alexander L. Ringer

\section{Nachbemerkung des Verlags}

Alexander L. Ringer ist am 2. Mai 2002 im Alter von 81 Jahren gestorben. Er hat das vorliegende Buch noch abgeschlossen; sein Erscheinen zu erleben war ihm verwehrt. Bei einem letzten Deutschland-Besuch habe ich ihn am 17. April 2002, zwei Wochen vor seinem Tod, in Frankfurt am Main getroffen. Bei dieser Gelegenheit sind wir alle noch offenen Fragen des Textes - endgültige Auswahl der Notenbeispiele, Nachweis von Anmerkungen und die Redaktion des Vorworts - durchgegangen. Was im Druck vorliegt, entspricht seinem letzten Willen. $\mathrm{Zu}$ danken habe ich Ringers amerikanischem Schüler Edward Hafer, der bei der Besorgung der Notenbeispiele behilflich war, Ullrich Scheideler von der Arbeitsstelle der Arnold-Schönberg-Gesamtausgabe, der das chronologische Werkverzeichnis nochmals überprüft hat, sowie Dr. Thomas Emmerig, der das Verzeichnis der zitierten Literatur sowie das Register erstellte.

Stuttgart, im Juni 2002

Uwe Schweikert

(J. B. Metzler Verlag) 
„Mit der Negation von Schein und Spiel tendiert Musik zur Erkenntnis.“ Theodor W. Adorno 